\title{
Transient Modulations of Neural Responses to Heartbeats Covary with Bodily Self-Consciousness
}

\author{
달eong-Dong Park, ${ }^{1}$ Fosco Bernasconi, ${ }^{1}$ Javier Bello-Ruiz, ${ }^{1}$ Christian Pfeiffer, ${ }^{1}$ Roy Salomon, ${ }^{1}$ and Olaf Blanke ${ }^{1,2}$ \\ ${ }^{1}$ Laboratory of Cognitive Neuroscience, Center for Neuroprosthetics and Brain Mind Institute, Ecole Polytechnique Fédérale de Lausanne, 1202 Geneva, \\ Switzerland, and 2Department of Neurology, University of Geneva, 1211 Geneva, Switzerland
}

Recent research has investigated self-consciousness associated with the multisensory processing of bodily signals (e.g., somatosensory, visual, vestibular signals), a notion referred to as bodily self-consciousness, and these studies have shown that the manipulation of bodily inputs induces changes in bodily self-consciousness such as self-identification. Another line of research has highlighted the importance of signals from the inside of the body (e.g., visceral signals) and proposed that neural representations of internal bodily signals underlie self-consciousness, which to date has been based on philosophical inquiry, clinical case studies, and behavioral studies. Here, we investigated the relationship of bodily self-consciousness with the neural processing of internal bodily signals. By combining electrical neuroimaging, analysis of peripheral physiological signals, and virtual reality technology in humans, we show that transient modulations of neural responses to heartbeats in the posterior cingulate cortex covary with changes in bodily self-consciousness induced by the full-body illusion. Additional analyses excluded that measured basic cardiorespiratory parameters or interoceptive sensitivity traits could account for this finding. These neurophysiological data link experimentally the cortical mapping of the internal body to selfconsciousness.

Key words: bodily self-consciousness; full-body illusion; heartbeat evoked potentials

Significance Statement

What are the brain mechanisms of self-consciousness? Prominent views propose that the neural processing associated with signals from the internal organs (such as the heart and the lung) plays a critical role in self-consciousness. Although this hypothesis dates back to influential views in philosophy and psychology (e.g., William James), definitive experimental evidence supporting this idea is lacking despite its recent impact in neuroscience. In the present study, we show that posterior cingulate activities responding to heartbeat signals covary with changes in participants' conscious self-identification with a body that were manipulated experimentally using virtual reality technology. Our finding provides important neural evidence about the long-standing proposal that self-consciousness is linked to the cortical processing of internal bodily signals.

\section{Introduction}

What are the neural mechanisms of self-consciousness (i.e., of the self as a subject of conscious experience)? Recent research on selfconsciousness has defined and investigated neuroscience-based concepts of self-consciousness (Ehrsson, 2007; Lenggenhager et al., 2007; Blanke and Metzinger, 2009; Blanke et al., 2015) and has high-

Received Jan. 28, 2016; revised June 22, 2016; accepted June 24, 2016.

Author contributions: H.-D.P. and 0.B. designed research; H.-D.P., F.B., J.B.-R., C.P., and R.S. performed research; H.-D.P. and F.B. analyzed data; H.-D.P., R.S., and O.B. wrote the paper.

This work was supported by the Fondation Bertarelli. We thank Andrea Serino, Aaron Schurger, and Marco Solca for useful comments.

The authors declare no competing financial interests.

Correspondence should be addressed to Dr. Olaf Blanke, Laboratory of Cognitive Neuroscience, Center for Neuroprosthetics and Brain Mind Institute, Ecole Polytechnique Fédérale de Lausanne (EPFL), 9 Chemin des Mines, 1202 Geneva, Switzerland. E-mail: olaf.blanke@epfl.ch.

DOI:10.1523/JNEUROSCI.0311-16.2016

Copyright $\odot 2016$ the authors $\quad 0270-6474 / 16 / 368453-08 \$ 15.00 / 0$ lighted the contributions of the multisensory processing of bodily stimuli (e.g., somatosensory, visual, vestibular, visceral signals), a notion referred to as bodily self-consciousness (Blanke et al., 2015). In these studies, experimental manipulations of the perception of multisensory bodily stimuli have been shown to induce changes in clearly defined aspects of bodily self-consciousness such as selfidentification or self-location (Ehrsson, 2007; Lenggenhager et al., 2007; Ionta et al., 2011; Petkova et al., 2011; Blanke et al., 2015). In the full-body illusion paradigm, for example, visuotactile stimulation is used by synchronously stroking the back of a participant coupled with viewed stroking applied to a virtual body and this has been shown repeatedly to induce increased self-identification with the virtual body compared with asynchronous stroking conditions (Lenggenhager et al., 2007; Ionta et al., 2011).

Although previous studies have focused on exteroceptive bodily signals such as visual and tactile inputs, other researchers have emphasized the importance of interoceptive signals in self- 


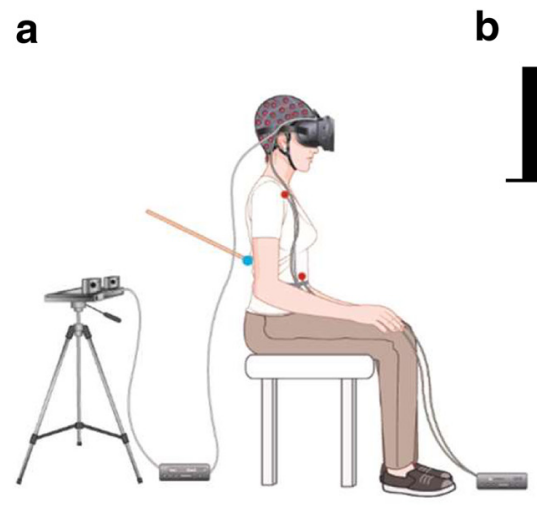

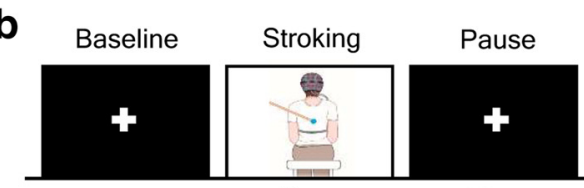

$100 \mathrm{sec} \quad \begin{gathered}40 \mathrm{sec} \quad 20 \mathrm{sec} \\ \quad \text { Repeated } 5 \text { times } \boldsymbol{4}\end{gathered}$

C

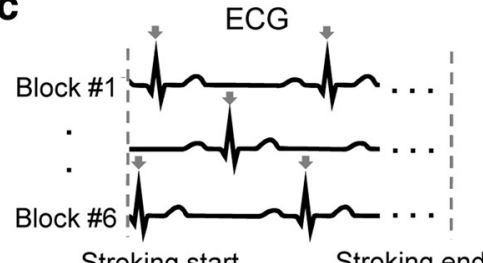

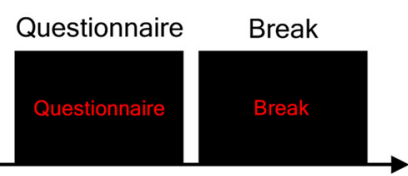

$120 \mathrm{sec}$

d

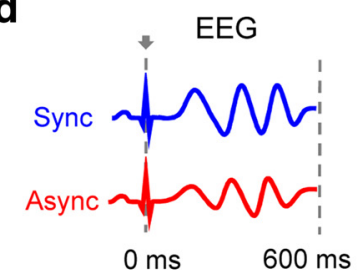

Figure 1. Experimental setup, procedure, and rationale. $\boldsymbol{a}$, We recorded EEG signals while participants' backs were stroked either synchronously or asynchronously with a virtually presented image of their own back through the head-mounted display. Two red dots on the trunk indicate the location of ECG electrodes. $\boldsymbol{b}$, Time course of a block. After five stroking-pause repetitions, participants were prompted to respond to the questionnaire. $\boldsymbol{c}$, Cardiac R-peaks (arrows) occurring during the stroking were detected in ECG signals in each block. $\boldsymbol{d}$, Neural responses to heartbeats during the stroking were obtained by averaging EEG signals locked on the ECG R-peaks (arrows) separately in synchronous and asynchronous conditions. The statistical analysis focused on the 0 - 600 ms post-R-peak time window without any a priori assumption.

consciousness and proposed that cortical representations of internal states of the body underlie the neural basis of self-consciousness (Craig, 2009; Christoff et al., 2011; Critchley and Harrison, 2013; Damasio and Carvalho, 2013; Seth, 2013; Park and Tallon-Baudry, 2014). The CNS constantly monitors vital internal organs, including the heart and the lung, for homeostatic regulation. Such neural processing of interoceptive signals has been proposed to provide continuity and stability for the organism and they have been argued to be indispensable for self-consciousness (Damasio, 2003; Critchley and Harrison, 2013; Park and Tallon-Baudry, 2014). Moreover, recent behavioral studies have shown functional links between interoceptive and exteroceptive signals in bodily self-consciousness. Therefore, multisensory stimulation involving cardiac and visual signals has been shown to affect the sense of hand ownership (Suzuki et al., 2013) and self-identification with the body (Aspell et al., 2013) and damage to the insula, a primary viscerosensory cortical region, has been shown to be associated with abnormal states of bodily selfconsciousness (Heydrich and Blanke, 2013; Ronchi et al., 2015). Based on these theoretical, behavioral, and clinical findings, we hypothesized that the neural processing of interoceptive signals would be linked to states of bodily self-consciousness as altered experimentally by the full-body illusion paradigm.

To test this hypothesis, we measured neural responses to heartbeats (Pollatos and Schandry, 2004; Gray et al., 2007; Park et al., 2014). Recent studies have shown that cardiac afferent signals and/or their neural processing can affect emotional appraisal (Gray et al., 2012; Garfinkel et al., 2014), visual awareness (Park et al., 2014; Salomon et al., 2016), memory encoding (Garfinkel et al., 2013), and somatosensory processing (Edwards et al., 2009; Gray et al., 2009). Neural processing of cardiac afferent signals can be measured noninvasively in humans via heartbeat-evoked potentials (HEPs), which are obtained by averaging electrophysiological signals time-locked to heartbeats. HEPs have been primarily observed over frontocentral regions in the time period ranging from 200 to $500 \mathrm{~ms}$ after the ECG R-peak (Pollatos and Schandry, 2004; Park et al., 2014). HEPs also have been associated with interoceptive functions such as heartbeat perception (Pollatos and Schandry, 2004) and cardiac output (Gray et al., 2007). Of particular relevance for the present study, Park et al. (2014) showed that the HEP amplitude affects conscious perception of exteroceptive stimuli (e.g., emotionally neutral visual gratings). In the present study, we investigated the relationship of HEPs with bodily self-consciousness. For this, we tested whether the HEP amplitude differs between two experimental conditions of the full-body illusion paradigm (e.g., synchronous and asynchronous visuotactile stimulation) that induce differential illusory states of bodily self-consciousness and whether such HEP amplitude modulations correlate with the questionnaire scores measuring the strength of illusory states.

\section{Materials and Methods}

Participants. Seventeen participants (4 female; 2 left-handed; mean age: $25.5 \pm 0.8$ years) with normal or corrected-to-normal vision took part in the study and reported no history of cardiovascular disease or neurological or psychiatric disorders. All subjects signed a written informed consent and were paid for their participation. All procedures were approved by the local ethics committee. One participant was excluded from analysis because $>50 \%$ of the ECG R-peaks could not be identified due to corrupted ECG signals. The data from 16 subjects were analyzed.

Experimental setup. Participants wore a head-mounted display $(640 \times$ 800 resolution, $110^{\circ}$ diagonal field of view; Oculus Rift Development kit; Oculus VR) on the top of the EEG cap (Fig. 1a). During the stroking, participants viewed an image of their own back in a sitting position through a head-mounted display from cameras (C510; Logitech) located $2 \mathrm{~m}$ behind their real body while the experimenter gave irregular stroking at velocity of $\sim 4 \mathrm{~cm} / \mathrm{s}$ on their real back using a stick with a blue pointer on the tip. The experimenter was blinded to the condition and participants were instructed to attend to the blue pointer of the stick on the virtually presented body image. During the synchronous condition, participants viewed their body image in real-time, whereas $0.5 \mathrm{~s}$ of delay was introduced during the asynchronous condition. Participants also wore a white t-shirt and ear plugs through the experiment. EXpyVR (custom in-house software, http://nco.epfl.ch/expyvr) was used for the visual stimulus presentation.

Procedure. Before the full-body illusion experiment, participants performed a heartbeat perception task (Schandry, 1981; Tsakiris et al., 2011). After a short training session (15 s), participants were instructed to silently count and report their heartbeats during five fixed time intervals $(25,35,45,60$, and $100 \mathrm{~s}$ in randomized order) without taking their pulse or feedback on their performance. Heartbeat perception score was calculated using the following formula (Tsakiris et al., 2011):

$$
\frac{1}{5} \Sigma\left(1-\frac{\mid \text { recorded heartbeats }- \text { counted heartbeats } \mid}{\text { recorded heartbeats }}\right)
$$

Participants then performed a brief training session consisting of $40 \mathrm{~s}$ of synchronous and asynchronous stroking in randomized order, followed 
by a questionnaire. The main experimental session (Fig. 1b) consisted of six blocks: three synchronous blocks and three asynchronous blocks in randomized order. Each block began with a baseline period in which participants fixated on a white cross on the center of view for $100 \mathrm{~s}$. Then, participants' backs were stroked either synchronously or asynchronously while viewing the virtual body for $40 \mathrm{~s}$. Then, a white cross appeared again for $20 \mathrm{~s}$ pause period. After five stroking-pause repetitions, participants were prompted to respond verbally to the questionnaire. Three questions were selected from the previous study (Ionta et al., 2011) to inquire about the state of participants' self-identification (Q1: How strong was the feeling that the body you saw was you?), illusory touch (Q2: How strong was the feeling that the touch you felt was located where you saw the stroking?), and for the control purpose (Q3: I felt my body as usual, nothing changed) using a 7-point scale ranging from 1 (bottomextreme) to 7 (top-extreme). To ensure their comprehension, participants were explicitly told by the experimenter that the "body" in Q3 indicates the participant's physical body, not the visually presented one. After $120 \mathrm{~s}$ of break, the next block began.

EEG recording and analysis. Continuous EEG signals were collected using a 64-channel active electrode EEG system (ActiveTwo system; BioSemi) at a sampling rate of $2048 \mathrm{~Hz}$ and online low-pass filtered at 400 $\mathrm{Hz}$. Bipolar ECG electrodes were placed over the top of the right shoulder and the bottom of the left side of the abdomen. Vertical and horizontal EOGs were recorded simultaneously.

Preprocessing and averaging were conducted using the Fieldtrip toolbox (Oostenveld et al., 2011). Continuous EEG and ECG data were down-sampled to $256 \mathrm{~Hz}$ and offline filtered between 1 and $40 \mathrm{~Hz}$. EEG data were re-referenced to a common average reference. Independent component analysis was conducted on the continuous EEG signals and stereotypical independent components reflecting eye movements and eye blinks were removed based on the visual inspection of all of the independent components (Delorme and Makeig, 2004). EEG signals of malfunctioning electrodes (median: 1, range: $0-2$ electrodes) were interpolated by computing average of neighboring electrodes.

HEPs were computed on EEG signals locked to the R-peak of the ECG (Fig. 1c,d). R-peaks were detected on ECG lead-II by correlating the ECG lead-II signal with a template QRS complex defined on a subject-bysubject basis and local maxima within episodes of correlation larger than 0.7 were identified. Then, single epochs were inspected visually to confirm that no artifacts remained. After artifact correction, $667 \pm 28$ and $679 \pm 26$ (mean \pm SEM) epochs were averaged to compute HEPs for synchronous and asynchronous conditions, respectively, during the stroking period.

Respiration recording and analysis. Continuous respiratory signals were recorded in subjects using a respiration belt (SleepSense 1387-kit; Sleep Laboratory Products) and offline filtered between 0.1 and $10 \mathrm{~Hz}$. Heartbeat-locked respiration was computed by averaging respiratory signals time locked to the R-peak separately in synchronous and asynchronous conditions.

Surrogate R-peaks. Surrogate R-peaks were created to investigate whether the differential HEP modulation is time locked to the original R-peaks. To create surrogate R-peaks that preserve the same interbeat interval and variability with the original R-peaks, the onset of all the original R-peaks were randomly shifted by the same amount $(-500 \sim$ $+500 \mathrm{~ms}$ ) separately for each stroking period (e.g., 15 stroking periods for each synchronous and asynchronous condition) and for each subject. A total of 200 sets of surrogate R-peaks were created.

Source localization. Source localization and surface visualization were performed with the BrainStorm toolbox (Tadel et al., 2011). Electrical cortical activity was estimated using a distributed model consisting of 15,000 current dipoles. The forward model was computed using the Open-MEEG Boundary Element Method (Gramfort et al., 2010) on the cortical surface of Colin27 MNI template. The sources of EEG signal were computed using a linear inverse estimator (weighted minimum-norm current estimate with dSPM option; depth weighting order: 0.5 , maximal amount: 10; signal-to-noise ratio: 3 ; noise covariance regularization: 0.1 ) with unconstrained dipole orientations separately for each condition and for each subject. Cortical currents were then averaged over the time window in which a significant difference between synchronous and asyn- chronous conditions was identified in the electrode level. The reliability of the difference in absolute dipole current was assessed by the clusterbased permutation $t$ test as detailed below. Anatomical description is based on the Desikan-Killiany parcellation (Desikan et al., 2006).

Statistical analysis. The significance of differences of HEPs between synchronous and asynchronous conditions was tested using the cluster based permutation $t$ test (Maris and Oostenveld, 2007) as implemented in the Fieldtrip toolbox (Oostenveld et al., 2011). Individual samples with $t$-values that exceed a threshold ( $p<0.05$, two-tailed) were clustered based on temporal and spatial adjacency. Each cluster defined in time and space by this procedure was assigned cluster-level statistics corresponding to the sum of the $t$-values of the samples belonging to that cluster. The type-I error rate was controlled by evaluating the maximum cluster-level statistics under the null hypothesis: condition labels were randomly shuffled 1000 times to estimate the distribution of maximal cluster-level statistics obtained by chance. The two-tailed Monte-Carlo $p$-value corresponds to the proportion of the elements in the distribution of shuffled maximal cluster-level statistics that exceeds the observed maximum or minimum original cluster-level test statistics. Because this method uses maxima, it corrected intrinsically for multiple comparisons in time and space. This procedure was applied at the sensor level in the time window from 0 to $600 \mathrm{~ms}$ after the R-peaks. The same procedure was applied at the source level on currents averaged between 250 and 305 $\mathrm{ms}$ across the 15,000 vertices of the cortical model (vertexwise threshold: $p<0.005$, two-tailed). It was also used for the mean HEP amplitudes analysis over the five time bins (baseline, three equally sized time bins of stroking, and pause period; see Fig. $3 d$ ) to correct for multiple comparisons and to find significant temporal clusters over the time bins.

Within-subject correlation analysis between the HEP amplitude and illusion rating (Fig. $3 e$ ) was evaluated statistically with a permutation test. For each subject, block labels were shuffled randomly separately for the mean HEP amplitudes and illusion rating, and Spearman's $\rho$ was computed following the same procedure used for the original data. Then, the grand average of Spearman's $\rho$ across 16 subjects was obtained with these shuffled data. This procedure was repeated 1000 times, producing a distribution of the grand averaged Spearman's $\rho$ s that can be observed by chance. A $p$-value was obtained as the proportion of the grand averaged Spearman's $\rho$ s from shuffled data that exceed the original grandaveraged Spearman's $\rho$.

To further confirm the results of control analyses regarding cardiorespiratory parameters (e.g., ECG amplitude, interbeat interval, heart rate variability, respiratory activity), a Bayesian paired $t$ test approach (Rouder et al., 2009) was applied. The inverse Bayes factors (e.g., JZS Bayes factor with scale factor $r=1$ ) were computed for those control analyses with the assumption that values $>3$ would support the null hypothesis, whereas values $<1 / 3$ would indicate evidence for the alternative hypothesis.

\section{Results}

\section{Questionnaire results}

As shown in Figure 2, increased self-identification (Q1) and illusory touch (Q2) for the virtually viewed body were obtained in the synchronous condition compared with the asynchronous condition (Wilcoxon signed-rank test, both $p<0.01$, Bonferroni corrected for three comparisons), whereas responses to the control question (Q3) did not differ between conditions (Wilcoxon signed-rank test, $p=0.33$, Bonferroni corrected for three comparisons). Across subjects, the difference of the mean scores between conditions for self-identification and illusory touch correlated with each other (Spearman's $\rho$ across subjects, $\rho=0.6$, $p=0.014)$, but neither of them correlated with the difference scores for the control question (both $\rho<0.4$, both $p>0.15$ ). These results demonstrate that the synchronous visuotactile manipulation induced changes in bodily self-consciousness, which is in agreement with previous findings (Lenggenhager et al., 2007; Ionta et al., 2011). 


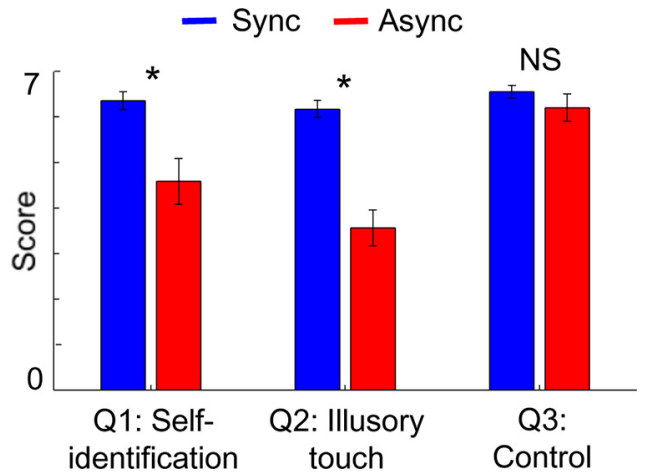

Figure 2. Questionnaire scores. Self-identification (Q1) and illusory touch (Q2) scores were higher in the synchronous compared with the asynchronous condition. Error bars indicate SEM. ${ }^{*} p<0.05$. NS, Not significant.

\section{Neural responses to heartbeats reflect bodily self-consciousness}

We tested the hypothesis that neural responses to heartbeats would reflect this altered state of bodily self-consciousness as induced by multisensory visuotactile stimulation. We averaged EEG signals time locked to the R-peak of the ECG and computed HEPs (Pollatos and Schandry, 2004; Gray et al., 2007) during the stroking period separately for both the synchronous and asynchronous conditions (Fig. $1 c, d$ ). We then submitted the $0-600$ ms post R-peak time window to a cluster-based permutation $t$ test (see Materials and Methods). The HEP amplitude differed significantly between the synchronous and asynchronous conditions (cluster-level $p=0.01$, corrected for multiple comparisons in space and time) over frontocentral regions in the $250-305 \mathrm{~ms}$
post-R-peak period (Fig. 3a,b). As shown in Figure $3 c$, the mean HEP amplitude that we obtained during the no-stroking period (e.g., all of the baseline and pause periods) was more positive than the mean HEP amplitude during the asynchronous condition (paired $t$ test, $t_{(15)}=-3.14, p=0.007$ ), but was more negative than the mean HEP amplitude during the synchronous condition $\left(t_{(15)}=3.35, p=0.004\right)$.

Importantly, we tested whether the difference in EEG signals as related to bodily self-consciousness was truly time locked to the heartbeat or if it reflected other changes in ongoing EEG activity that are not specifically associated with heartbeats (e.g., stroking-related sensory evoked components). We conducted the same HEP analysis repeatedly (200 times) using surrogate R-peaks, which had the same mean interval and variability as real $\mathrm{R}$-peaks but were shifted randomly in time (see Materials and Methods). We did not find a cluster $t$-statistic equal to or greater than the one initially obtained from the HEP with real R-peaks, confirming that the differential HEP effect is truly locked to the heartbeat (Monte-Carlo $p=0.005$ ).

Next, to investigate how the differential HEP evolved before, during, and after the time of multisensory visuotactile stimulation, we computed the difference of the mean HEP amplitudes between the two experimental conditions over the following five time bins: baseline, three equally sized time bins of stroking, and pause period (Fig. 3d). The HEP amplitudes differed between synchronous and asynchronous conditions in each of the three stroking period time bins (cluster-based permutation $t$ test over the five time bins, cluster-level $p=0.002$ ), but did not differ among them (one-way repeated-measures ANOVA over the three stroking period time bins, $\left.F_{(2,30)}=0.2, p=0.82\right)$. Such HEP

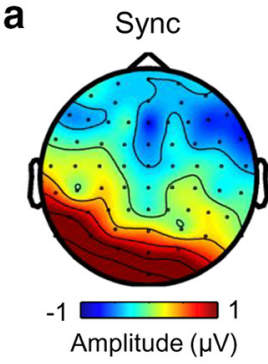

C

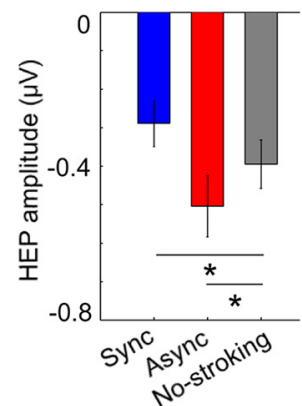

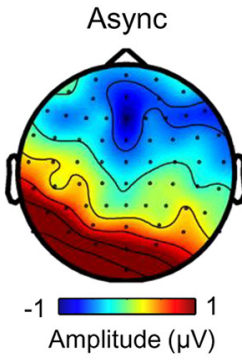

d

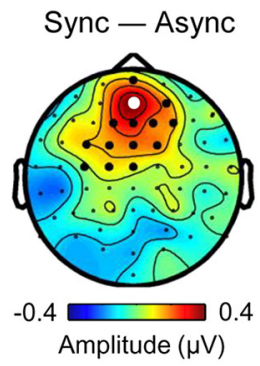

b

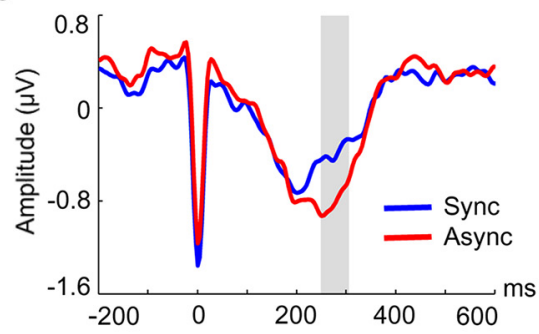

e

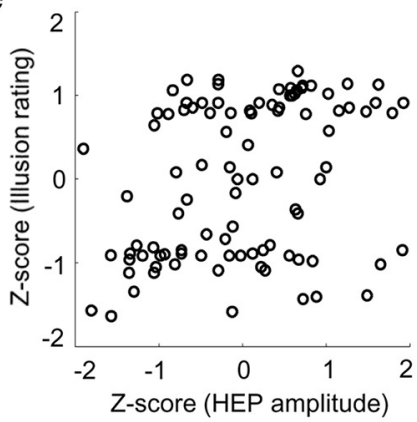

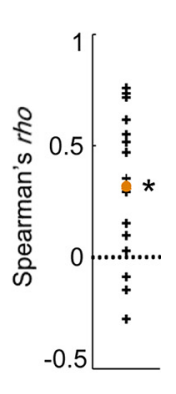

NS

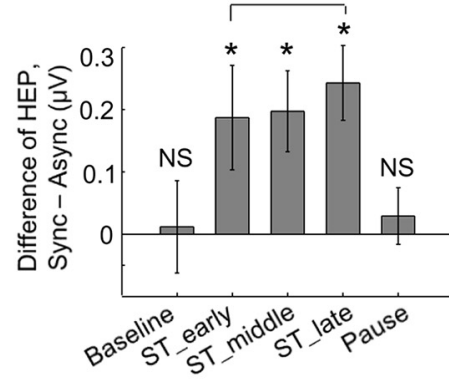

Figure 3. Neural responses to heartbeats reflect altered state of bodily self-consciousness. $\boldsymbol{a}$, Topographical map of the HEP amplitude during the synchronous condition (left), asynchronous condition (middle), and their differences (Sync - Async; right) in the 250-305 ms time window in which a significant difference is observed. Larger black and white dots indicate the electrodes contributing to the significant cluster. $\boldsymbol{b}$, Time course of HEPs at the electrode indicated by a white dot in $\boldsymbol{a}$, which showed maximal difference. The shaded area highlights the time window in which a significant difference is observed (cluster-level $p=0.01, n=16$ ). $c$, Mean HEP amplitude averaged across the cluster during the synchronous and asynchronous conditions and during the no-stroking period (e.g., all the baseline and pause periods). $\boldsymbol{d}$, Differences of the mean HEP amplitudes between synchronous and asynchronous conditions during the baseline, three equally sized time bins of stroking, and pause period. Differential HEP amplitudes were observed in each of the three stroking period time bins (all $p<0.05, n=16$ ), but not in the baseline or pause period (both $p>0.4, n=16)$. $\boldsymbol{e}$, Illusion rating as a function of the mean HEP amplitude across blocks in each subject (left). Each dot indicates a block. Spearman's $\rho$ in each subject is shown on the right. Each cross indicates a subject and the orange dot indicates the grand-averaged Spearman's $\rho$ across 16 subjects, which was significantly larger than 0 (permutation test, $p=0.004, n=16$ ). Error bars indicate SEM. * $p<0.05$. NS, Not significant. 
differences were not observed in the baseline or the pause period ( $t$ test against 0 , both $p>0.4$ ). This pattern of results indicates that synchronous multisensory stimulation transiently induced the observed changes in HEPs.

We then tested whether the neural responses to heartbeats correlated with the subjective effects of the illusion. To this aim, in each subject and block, we computed the mean HEP amplitude and illusion rating (mean score of Q1 and Q2). For each subject, these raw values in each block were transformed into $Z$-scores using the mean and the SD obtained across six blocks $[Z$-score $=$ (raw value - mean)/SD]. We then computed Spearman's $\rho$ between the HEP amplitude and the illusion rating using these $Z$-scores in each subject. Across subjects, Spearman's $\rho$ s were significantly larger than 0 (Fig. $3 e$; mean $\rho, 0.32 \pm 0.08$; permutation test, $p=0.004)$. No such correlation was found for the control question $(\mathrm{Q} 3$, mean $\rho=-0.13 \pm 0.11 ; p=0.38)$.

Next, we investigated whether these HEP effects were related to our participants' explicit sensitivity to interoceptive signals as measured by the heartbeat perception task (Tsakiris et al., 2011; see Materials and Methods). However, this was not the case: the heartbeat perception scores $(0.70 \pm 0.03$, mean \pm SEM $)$ did not correlate with the differences of the mean HEP amplitudes ( $\rho=$ $-0.05, p=0.8$ ) or questionnaire scores (all $p>0.3$ ). This is compatible with the transient nature of the self-related HEP modulation (Fig. $3 d$ ) and suggests that the modulation of HEP reflects the transient online character of our participants' altered state rather than their persistent offline interoceptive sensitivity traits.

To identify the cortical sources of the differential HEPs observed over the frontocentral scalp regions, we reconstructed the neural sources of the EEG signals separately in synchronous and asynchronous conditions (Fig. 4). We found that the bilateral posterior cingulate cortex (PCC) extending to the supplementary motor area (Table 1) were activated differentially (cluster-based permutation $t$ test in space on mean 250-305 ms currents, both cluster-level $p<0.05$ ). At a lower threshold (vertexwise $p<$ 0.05 ), we observed the third largest cluster next to the right and left (bilateral) PCC clusters at the left insular cortex (cluster size $=112$ vertices), a known cortical target of cardiac afferent signals (Craig, 2009; Critchley and Harrison, 2013); however, this region did not survive multiple comparison correction.

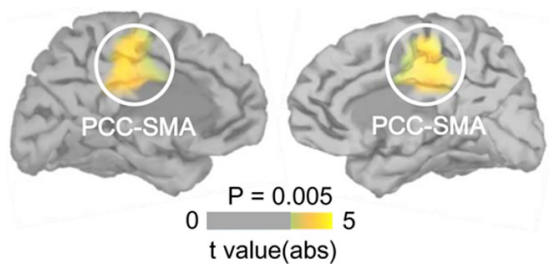

Figure 4. Neural sources of the differential HEP. Shown is the differential activation by heartbeats in bilateral $\mathrm{PCC}$ between synchronous and asynchronous conditions during the stroking period (both cluster-level $p<0.05, n=16$ ).

Table 1. MNI coordinates ( $\mathrm{mm}$ ) of differential HEPs

\begin{tabular}{llllll}
\hline Region & $x$ & $y$ & $z$ & $\begin{array}{l}\text { Cluster size } \\
\text { (vertices) }\end{array}$ & Anatomical description \\
\hline Left PCC-SMA & -7 & -18 & 50 & 151 & $\begin{array}{c}\text { Dorsal posterior cingulate } \\
\text { extending into superior } \\
\text { frontal gyrus }\end{array}$ \\
Right PCC-SMA & 1 & -17 & 54 & 143 & $\begin{array}{c}\text { Dorsal posterior cingulate } \\
\text { extending into superior } \\
\text { frontal gyrus }\end{array}$ \\
\hline
\end{tabular}

\section{Cardiorespiratory parameters}

We performed extensive control analyses concerning measured cardiorespiratory parameters that could have confounded the present HEP results. First, we tested whether the differential HEP amplitudes recorded from the scalp EEG were due to artifacts from the cardiac field (Dirlich et al., 1997). This was not the case: the ECG data almost completely overlapped in both conditions (Fig. $5 a$ ) and did not show any difference in the time window in which the differential HEP was observed (paired $t$ test on the mean 250-305 ms ECG amplitudes, $t_{(15)}=0.37, p=0.71$; Bayesian paired $t$ test, inverse Bayes factor $=4.95$ ). Moreover, measured basic cardiac parameters did not differ between conditions: the interbeat interval and heart rate variability (Fig. $5 c$ ) did not differ between conditions (all $p>0.3$; all inverse Bayes factors $>$ 3.5). Next, we investigated the possibility that differential HEP effects could be associated with changes in respiratory activity. As shown in Figure $5 b$, we analyzed heartbeat-locked respiratory activity, but did not find any differences between synchronous and asynchronous conditions in the time window in which the differential HEP was observed (paired $t$ test on the mean 250-305 ms respiratory activity, $t_{(15)}=0.007, p=0.99$; Bayesian paired $t$ test, inverse Bayes factor $=5.29$ ).

\section{Discussion}

We found that transient modulations of neural activation in the PCC locked to heartbeats covary with experimentally induced altered states of bodily self-consciousness. Moreover, the HEP modulation correlated with participants' subjective illusion effects and did not relate to any of the other measured cardiorespiratory parameters or interoceptive sensitivity traits. The present findings provide neurophysiological evidence supporting the proposed relationship between the brain's mapping of the internal body and self-consciousness, which to date has primarily been based on speculation (Damasio, 2003; Christoff et al., 2011; Park and Tallon-Baudry, 2014), clinical case studies (Critchley and Harrison, 2013; Damasio and Carvalho, 2013; Heydrich and Blanke, 2013; Ronchi et al., 2015), and behavioral studies (Aspell et al., 2013; Suzuki et al., 2013).

Our results are consistent with previous work on neural responses to heartbeats and known physiological pathways of viscerosensory signals. First, the observed differential neural responses to heartbeats are consistent with the previous HEP literature that found differential modulation between 200 and $500 \mathrm{~ms}$ after the R-peak over frontocentral regions (Pollatos and Schandry, 2004; Park et al., 2014). Second, we identified the neural sources of the differential self-related HEP in the PCC. Internal bodily signals are relayed to the neocortex primarily targeting the insula, cingulate cortex, somatosensory cortex, and amygdala (Craig, 2009; Critchley and Harrison, 2013; Damasio and Carvalho, 2013). Among the different cingulate subregions (e.g., anterior, middle, posterior cingulate), the anterior cingulate cortex (ACC) has traditionally been described as the main viscerosensory region (Vogt, 2005). However, more recent animal studies showed that direct stimulation of visceral organs evokes neural activity, not only in the ACC, but also in the PCC (Sikes et al., 2008), which is consistent with human neuroimaging data reporting the association between the BOLD response in the PCC and heartbeat-related processing (Critchley et al., 2003; Wong et al., 2007).

Our findings were further corroborated by additional analyses testing potential alternative explanations for our findings. First, the effect might reflect changes in ongoing EEG activity that is not specifically associated with heartbeats. Our analysis excluded this account: when the same EEG signals were locked to randomly 
shifted R-peaks, the effect could not be observed, indicating that the HEP effect is truly time locked to the original R-peaks. Second, the HEP effect might reflect heart electrical activity (e.g., cardiac field artifact; Dirlich et al., 1997), respiratory activity, or changes in the strength of cardiac input. However, measured basic cardiorespiratory parameters (e.g., ECG amplitude, heart rate, heart rate variability, respiratory activity) did not differ between conditions. Although this confirms that the HEP effect reflects changes in brain activity time locked to heartbeats rather than cardiac field artifact or respiratory activity, the relationship between HEPs and additional cardiac parameters should be investigated in future studies because we did not measure other cardiac parameters (e.g., stroke volume; Park et al., 2014; Winston and Rees, 2014) in the present study. Finally, it could be argued that the HEP effect might be associated with participants' interoceptive sensitivity. However, our data do not support this possibility: neither changes in HEP amplitudes nor changes in questionnaire scores correlated with heartbeat perception score, which is an important marker of interoceptive ability.

Recent behavioral studies have shown that the manipulation of multisensory stimulation using integrated cardiac and visual stimulation modulates the sense of hand ownership (Suzuki et al., 2013) and self-identification (Aspell et al., 2013), suggesting that interoceptive signals are another source of multisensory bodily input contributing to bodily self-consciousness. The chosen approach in the present study was different: using visuotactile stimulation, we showed that neural processing of interoceptive signals is associated with the strength of subjective state of bodily selfconsciousness even without the manipulation of the cardiac signal itself. In the current study, we decided to use visuotactile stimulation (rather than cardiovisual stimulation) because, when visual stimuli are time locked to ECG R-peaks (as in the previous cardiovisual experiments, e.g., Aspell et al., 2013; Suzuki et al., 2013), it is difficult to dissociate the influence of heartbeat-locked visual evoked potentials from heartbeat evoked potentials in the EEG data.

Our findings suggest that not only the neural processing of exteroceptive bodily signals, but also neural responses to interoceptive signals contribute to the brain mechanisms of bodily selfconsciousness (for clinical evidence, see Heydrich and Blanke, 2013; Ronchi et al., 2015). Previous fMRI studies found that activation in the premotor cortex, intraparietal sulcus regions, and at the temporoparietal junction is associated with illusory effects during different full-body illusion paradigms (Ionta et al., 2011; Petkova et al., 2011; Guterstam et al., 2015) and suggested that multisensory integration of exteroceptive bodily signals (e.g., visual, tactile, proprioceptive signals) in these regions is associated with key aspects of bodily self-consciousness, such as selfidentification and full-body ownership. Here, we report that the HEP amplitude positively correlates with illusion rating, comparable to previous reports showing that fMRI activity in the premotor cortex correlates with the illusory body ownership (Petkova et al., 2011; Guterstam et al., 2015). However, to our b
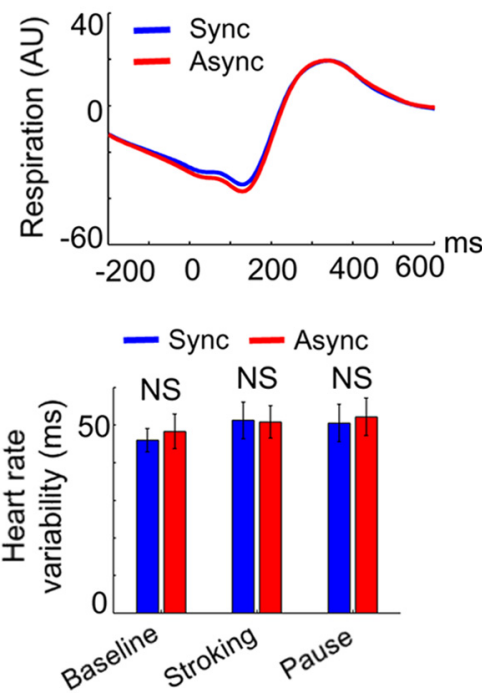

Figure 5. ECG amplitude, interbeat interval, heart rate variability, and respiratory activity did not differ between conditions. $\boldsymbol{a}$, R-peak-locked ECG signals during the stroking. No difference between synchronous and asynchronous conditions was observed (all te variability (right) during the baseline, stroking, and pause periods. No difference between synchronous and asynchronous

knowledge, little is known about the interaction between exteroceptive and interoceptive signals at the neural level. At least two mechanisms (that are not mutually exclusive) could potentially underlie integration of information encoded by interoceptive and exteroceptive neural systems (Park and Tallon-Baudry, 2014). The first possibility is that neural processing of interoceptive and exteroceptive signals converge onto common multisensory areas in a hierarchical structure. Indeed, recent theoretical models have proposed that the insula and cingulate cortex are involved in the construction of such a multisensory representation of the body (and self) integrating both exteroceptive and interoceptive signals (Craig, 2009; Heydrich and Blanke, 2013; Seth, 2013; Ronchi et al., 2015). The other possibility is that activity in exteroceptive multisensory areas and the neural processing of interoceptive signals are coordinated through interarea communication mechanisms such as oscillatory synchrony (Varela et al., 2001; Fries, 2005). These two mechanisms are not mutually exclusive. Indeed, recently, it has been proposed that the insula and the cingulate cortex can function as "rich-club" hubs and contribute to the construction of the widely distributed multisensory representation system through neural communication (Barrett and Simmons, 2015; Allen et al., 2016). In addition, caution should be exercised not to overinterpret our findings. Because the methods used in the present study were correlative in nature, our results cannot demonstrate any causal relationship between the HEP and changes in bodily self-consciousness. For instance, our data cannot reveal whether the observed differential HEP modulation caused changes in bodily self-consciousness or vice versa.

We further propose that the present data are of relevance beyond bodily self-consciousness because a recent study has shown that neural responses to heartbeats before stimulus onset can predict the subsequent conscious detection of a visual grating at threshold (Park et al., 2014). These investigators proposed that neural representations of afferent visceral signals carry information related to the subjectivity of perceptual conscious experience. However, in 
combination with the present data, the neural representations of afferent visceral information may not only be relevant for perceptual awareness of external stimuli, but also for self-consciousness. The findings reported here lend support to such a stronger hypothesis (Park and Tallon-Baudry, 2014) by linking neural responses to heartbeats to changes in subjective states of bodily self-consciousness, further strengthened by the known role of the PCC in mental states related to subjective experiences, including self-related processing (Northoff et al., 2006; Beer, 2007; Peer et al., 2015), selfreferential judgment (Whitfield-Gabrieli et al., 2011), subjective value rating (Kable and Glimcher, 2007), and the sense of selflocation (Guterstam et al., 2015).

To conclude, we demonstrate here the transient relationship between neural events time locked to heartbeats and illusory states of bodily self-consciousness. Our findings provide neural evidence to the previously proposed link between the brain's processing of visceral signals and self-consciousness. Together with a recent report that neural responses to heartbeats shape conscious visual perception (Park et al., 2014), our findings further suggest that neural processing of internal states of the body is a fundamental biological mechanism for the subjective dimension of conscious experience including, not just perceptual awareness, but also self-consciousness.

\section{References}

Allen M, Fardo F, Dietz MJ, Hillebrandt H, Friston KJ, Rees G, Roepstorff A (2016) Anterior insula coordinates hierarchical processing of tactile mismatch responses. Neuroimage 127:34-43. CrossRef Medline

Aspell JE, Heydrich L, Marillier G, Lavanchy T, Herbelin B, Blanke O (2013) Turning body and self inside out: visualized heartbeats alter bodily selfconsciousness and tactile perception. Psychol Sci 24:2445-2453. CrossRef Medline

Barrett LF, Simmons WK (2015) Interoceptive predictions in the brain. Nat Rev Neurosci 16:419-429. CrossRef Medline

Beer JS (2007) The default self: feeling good or being right? Trends Cogn Sci 11:187-189. CrossRef Medline

Blanke O, Metzinger T (2009) Full-body illusions and minimal phenomenal selfhood. Trends Cogn Sci 13:7-13. CrossRef Medline

Blanke O, Slater M, Serino A (2015) Behavioral, neural, and computational principles of bodily self-consciousness. Neuron $88: 145-166$. CrossRef Medline

Christoff K, Cosmelli D, Legrand D, Thompson E (2011) Specifying the self for cognitive neuroscience. Trends Cogn Sci 15:104-112. CrossRef Medline

Craig AD (2009) How do you feel-now? The anterior insula and human awareness. Nat Rev Neurosci 10:59-70. CrossRef Medline

Critchley HD, Harrison NA (2013) Visceral influences on brain and behavior. Neuron 77:624-638. CrossRef Medline

Critchley HD, Mathias CJ, Josephs O, O’Doherty J, Zanini S, Dewar BK, Cipolotti L, Shallice T, Dolan RJ (2003) Human cingulate cortex and autonomic control: converging neuroimaging and clinical evidence. Brain 126:2139-2152. CrossRef Medline

Damasio A (2003) Mental self: The person within. Nature 423:227. CrossRef Medline

Damasio A, Carvalho GB (2013) The nature of feelings: evolutionary and neurobiological origins. Nat Rev Neurosci 14:143-152. CrossRef Medline

Delorme A, Makeig S (2004) EEGLAB: an open source toolbox for analysis of single-trial EEG dynamics including independent component analysis. J Neurosci Methods 134:9-21. CrossRef Medline

Desikan RS, Ségonne F, Fischl B, Quinn BT, Dickerson BC, Blacker D, Buckner RL, Dale AM, Maguire RP, Hyman BT, Albert MS, Killiany RJ (2006) An automated labeling system for subdividing the human cerebral cortex on MRI scans into gyral based regions of interest. Neuroimage 31: 968-980. CrossRef Medline

Dirlich G, Vogl L, Plaschke M, Strian F (1997) Cardiac field effects on the EEG. Electroencephalogr Clin Neurophysiol 102:307-315. CrossRef Medline

Edwards L, Ring C, McIntyre D, Winer JB, Martin U (2009) Sensory detection thresholds are modulated across the cardiac cycle: evidence that cu- taneous sensibility is greatest for systolic stimulation. Psychophysiology 46:252-256. CrossRef Medline

Ehrsson HH (2007) The experimental induction of out-of-body experiences. Science 317:1048. CrossRef Medline

Fries P (2005) A mechanism for cognitive dynamics: neuronal communication through neuronal coherence. Trends Cogn Sci 9:474-480. CrossRef Medline

Garfinkel SN, Barrett AB, Minati L, Dolan RJ, Seth AK, Critchley HD (2013) What the heart forgets: Cardiac timing influences memory for words and is modulated by metacognition and interoceptive sensitivity. Psychophysiology 50:505-512. CrossRef Medline

Garfinkel SN, Minati L, Gray MA, Seth AK, Dolan RJ, Critchley HD (2014) Fear from the heart: sensitivity to fear stimuli depends on individual heartbeats. J Neurosci 34:6573-6582. CrossRef Medline

Gramfort A, Papadopoulo T, Olivi E, Clerc M (2010) OpenMEEG: opensource software for quasistatic bioelectromagnetics. Biomed Eng Online 9:45. CrossRef Medline

Gray MA, Taggart P, Sutton PM, Groves D, Holdright DR, Bradbury D, Brull D, Critchley HD (2007) A cortical potential reflecting cardiac function. Proc Natl Acad Sci U S A 104:6818-6823. CrossRef Medline

Gray MA, Rylander K, Harrison NA, Wallin BG, Critchley HD (2009) Following one's heart: cardiac rhythms gate central initiation of sympathetic reflexes. J Neurosci 29:1817-1825. CrossRef Medline

Gray MA, Beacher FD, Minati L, Nagai Y, Kemp AH, Harrison NA, Critchley HD (2012) Emotional appraisal is influenced by cardiac afferent information. Emotion 12:180-191. CrossRef Medline

Guterstam A, Björnsdotter M, Gentile G, Ehrsson HH (2015) Posterior cingulate cortex integrates the senses of self-location and body ownership. Curr Biol 25:1416-1425. CrossRef Medline

Heydrich L, Blanke O (2013) Distinct illusory own-body perceptions caused by damage to posterior insula and extrastriate cortex. Brain 136: 790-803. CrossRef Medline

Ionta S, Heydrich L, Lenggenhager B, Mouthon M, Fornari E, Chapuis D, Gassert R, Blanke O (2011) Multisensory mechanisms in temporoparietal cortex support self-location and first-person perspective. Neuron 70:363-374. CrossRef Medline

Kable JW, Glimcher PW (2007) The neural correlates of subjective value during intertemporal choice. Nat Neurosci 10:1625-1633. CrossRef Medline

Lenggenhager B, Tadi T, Metzinger T, Blanke O (2007) Video ergo sum: manipulating bodily self-consciousness. Science 317:1096-1099. CrossRef Medline

Maris E, Oostenveld R (2007) Nonparametric statistical testing of EEG- and MEG-data. J Neurosci Methods 164:177-190. CrossRef Medline

Northoff G, Heinzel A, de Greck M, Bermpohl F, Dobrowolny H, Panksepp J (2006) Self-referential processing in our brain-a meta-analysis of imaging studies on the self. Neuroimage 31:440-457. CrossRef Medline

Oostenveld R, Fries P, Maris E, Schoffelen JM (2011) FieldTrip: Open source software for advanced analysis of MEG, EEG, and invasive electrophysiological data. Comput Intell Neurosci 2011:156869. CrossRef Medline

Park HD, Tallon-Baudry C (2014) The neural subjective frame: from bodily signals to perceptual consciousness. Philos Trans R Soc Lond B Biol Sci 369:20130208. CrossRef Medline

Park HD, Correia S, Ducorps A, Tallon-Baudry C (2014) Spontaneous fluctuations in neural responses to heartbeats predict visual detection. Nat Neurosci 17:612-618. CrossRef Medline

Peer M, Salomon R, Goldberg I, Blanke O, Arzy S (2015) Brain system for mental orientation in space, time, and person. Proc Natl Acad Sci U S A 112:11072-11077. CrossRef Medline

Petkova VI, Björnsdotter M, Gentile G, Jonsson T, Li TQ, Ehrsson HH (2011) From part- to whole-body ownership in the multisensory brain. Curr Biol 21:1118-1122. CrossRef Medline

Pollatos O, Schandry R (2004) Accuracy of heartbeat perception is reflected in the amplitude of the heartbeat-evoked brain potential. Psychophysiology 41:476-482. CrossRef Medline

Ronchi R, Bello-Ruiz J, Lukowska M, Herbelin B, Cabrilo I, Schaller K, Blanke O (2015) Right insular damage decreases heartbeat awareness and alters cardio-visual effects on bodily self-consciousness. Neuropsychologia 70 : 11-20. CrossRef Medline

Rouder JN, Speckman PL, Sun D, Morey RD, Iverson G (2009) Bayesian t tests for accepting and rejecting the null hypothesis. Psychon Bull Rev 16:225-237. CrossRef Medline

Salomon R, Ronchi R, Dönz J, Bello-Ruiz J, Herbelin B, Martet R, Faivre N, 
Schaller K, Blanke O (2016) The insula mediates access to awareness of visual stimuli presented synchronously to the heartbeat. J Neurosci 36 : 5115-5127. CrossRef Medline

Schandry R (1981) Heart beat perception and emotional experience. Psychophysiology 18:483-488. CrossRef Medline

Seth AK (2013) Interoceptive inference, emotion, and the embodied self. Trends Cogn Sci 17:565-573. CrossRef Medline

Sikes RW, Vogt LJ, Vogt BA (2008) Distribution and properties of visceral nociceptive neurons in rabbit cingulate cortex. Pain 135:160-174. CrossRef Medline

Suzuki K, Garfinkel SN, Critchley HD, Seth AK (2013) Multisensory integration across exteroceptive and interoceptive domains modulates selfexperience in the rubber-hand illusion. Neuropsychologia 51:2909-2917. CrossRef Medline

Tadel F, Baillet S, Mosher JC, Pantazis D, Leahy RM (2011) Brainstorm: a user-friendly application for MEG/EEG analysis. Comput Intell Neurosci 2011:879716. CrossRef Medline

Tsakiris M, Tajadura-Jiménez A, Costantini M (2011) Just a heartbeat away from one's body: interoceptive sensitivity predicts malleability of body-representations. Proc Biol Sci 278:2470-2476. CrossRef Medline

Varela F, Lachaux JP, Rodriguez E, Martinerie J (2001) The brainweb: phase synchronization and large-scale integration. Nat Rev Neurosci 2: 229-239. CrossRef Medline

Vogt BA (2005) Pain and emotion interactions in subregions of the cingulate gyrus. Nat Rev Neurosci 6:533-544. Medline

Whitfield-Gabrieli S, Moran JM, Nieto-Castañón A, Triantafyllou C, Saxe R, Gabrieli JD (2011) Associations and dissociations between default and self-reference networks in the human brain. Neuroimage 55:225-232. CrossRef Medline

Winston JS, Rees G (2014) Following your heart. Nat Neurosci 17:482-483. CrossRef Medline

Wong SW, Massé N, Kimmerly DS, Menon RS, Shoemaker JK (2007) Ventral medial prefrontal cortex and cardiovagal control in conscious humans. Neuroimage 35:698-708. CrossRef Medline 